OPEN ACCESS

Edited by:

Qiang Wang,

Institute of Hydrobiology (CAS), China

Reviewed by:

Yu-Zhong Zhang,

Shandong University, China

Qingfang $\mathrm{He}$,

University of Arkansas at Little Rock,

United States

*Correspondence: Jizhong Zhou jzhou@ou.edu

Specialty section:

This article was submitted to Microbiotechnology, Ecotoxicology

and Bioremediation,

a section of the journal

Frontiers in Microbiology

Received: 06 June 2017

Accepted: 28 August 2017 Published: 07 September 2017

Citation:

X U T, Li Y, He Z, Van Nostrand JD and Zhou J (2017) Cas9 Nickase-Assisted RNA Repression

Enables Stable and Efficient Manipulation of Essential Metabolic Genes in Clostridium cellulolyticum.

Front. Microbiol. 8:1744.

doi: 10.3389/fmicb.2017.01744

\section{Cas9 Nickase-Assisted RNA Repression Enables Stable and Efficient Manipulation of Essential Metabolic Genes in Clostridium cellulolyticum}

\author{
Tao Xu' ${ }^{1}$, Yongchao $\mathrm{Li}^{1}$, Zhili He ${ }^{1}$, Joy D. Van Nostrand ${ }^{1}$ and Jizhong Zhou 1,2,3* \\ 1 Institute for Environmental Genomics and Department of Microbiology and Plant Biology, University of Oklahoma, Norman, \\ OK, United States, ${ }^{2}$ Earth Sciences Division, Lawrence Berkeley National Laboratory, Berkeley, CA, United States, ${ }^{3}$ State \\ Key Joint Laboratory of Environment Simulation and Pollution Control, School of Environment, Tsinghua University, Beijing, \\ China
}

Essential gene functions remain largely underexplored in bacteria. Clostridium cellulolyticum is a promising candidate for consolidated bioprocessing; however, its genetic manipulation to reduce the formation of less-valuable acetate is technically challenging due to the essentiality of acetate-producing genes. Here we developed a Cas9 nickase-assisted chromosome-based RNA repression to stably manipulate essential genes in C. cellulolyticum. Our plasmid-based expression of antisense RNA (asRNA) molecules targeting the phosphotransacetylase (pta) gene successfully reduced the enzymatic activity by $35 \%$ in cellobiose-grown cells, metabolically decreased the acetate titer by 15 and $52 \%$ in wildtype transformants on cellulose and xylan, respectively. To control both acetate and lactate simultaneously, we transformed the repression plasmid into lactate production-deficient mutant and found the plasmid delivery reduced acetate titer by more than 33\%, concomitant with negligible lactate formation. The strains with pta gene repression generally diverted more carbon into ethanol. However, further testing on chromosomal integrants that were created by double-crossover recombination exhibited only very weak repression because DNA integration dramatically lessened gene dosage. With the design of a tandem repetitive promoter-driven asRNA module and the use of a new Cas9 nickase genome editing tool, a chromosomal integrant (LM3P) was generated in a single step and successfully enhanced RNA repression, with a $27 \%$ decrease in acetate titer on cellulose in antibioticfree medium. These results indicate the effectiveness of tandem promoter-driven RNA repression modules in promoting gene repression in chromosomal integrants. Our combinatorial method using a Cas9 nickase genome editing tool to integrate the gene repression module demonstrates easy-to-use and high-efficiency advantages, paving the way for stably manipulating genes, even essential ones, for functional characterization and microbial engineering.

Keywords: essential genes, genome editing, gene repression, metabolic engineering, consolidated bioprocessing, Clostridium cellulolyticum 


\section{INTRODUCTION}

Essential genes are indispensable for building up the chassis of living organisms (Glass et al., 2006), and accounts for 5-80\% of bacterial genomes (Gao and Zhang, 2011). Investigation into these genes will provide insights on basic biological functions and allow for the discovery of cellular activities with industrial or biomedical potentials (Lee et al., 2009; Juhas et al., 2012). Since genetic knock-outs of essential genes are lethal and then unobtainable (Glass et al., 2006), knock-down strategies are applicable and widely used (Ji et al., 1999). There are three major approaches available for targeted gene repression in bacteria, including antisense RNA (asRNA)-mediated repression (Desai and Papoutsakis, 1999; Perret et al., 2004; Thomason and Storz, 2010), Hfp-dependent RNA repression (Man et al., 2011; Na et al., 2013) and nuclease-null Cas9-mediated repression (which is named CRISPRi) (Bikard et al., 2013; Qi et al., 2013). The latter two require an RNA binding protein, Hfp chaperone and non-catalytic Cas 9 endonuclease, respectively, which need to be consistently co-expressed with RNA molecules that recognize target transcripts. Plasmid-based expression of these components has been widely applied in bacteria (Desai and Papoutsakis, 1999; Perret et al., 2004; Thomason and Storz, 2010; Man et al., 2011; Bikard et al., 2013; Na et al., 2013); however, concerns are raised about the stability and antibiotic dependence of plasmidbased expression (Lee et al., 1993; Friehs, 2004), especially in industrial microorganisms, and potential side effects caused by the specificity of RNA binding proteins (Martinez-Alonso et al., 2010; Bikard et al., 2013; Qi et al., 2013). Development of a relatively clean, easy and efficient approach allowing for rapidly generating stable knock-down mutants would increase our ability to study and manipulate essential genes. Considering the easy-to-use and highly efficient advantages of CRISPR/Cas9based genome editing tools ( $\mathrm{Xu}$ et al., 2014, 2015) and the simplicity and universality of antisense RNA-mediated repression (Thomason and Storz, 2010), here we propose a combination of these two methods using Cas9 technology to integrate antisense RNA modules into the genome. By doing so, knock-down mutants can be created in a single step with features that are plasmid-independent and can be sustained without using antibiotics.

Clostridium cellulolyticum H10, a model organism of mesophilic cellulolytic Clostridia, is an excellent consolidated bioprocessing host (Desvaux, 2005; Lynd et al., 2005). It can hydrolyze lignocellulose without adding commercial cellulases and simultaneously ferment a variety of C5 and C6 sugars to end products (lactate, acetate and ethanol) (Desvaux, 2005). Metabolic engineering significantly improved microbial characteristics via overexpressing foreign genes of intended pathways (Guedon et al., 2002; Higashide et al., 2011; Li et al., 2014; Lin et al., 2015), or eliminating competing and promiscuous pathways (Shaw et al., 2008; Li et al., 2012; Papanek et al., 2015). In C. cellulolyticum, a double mutation of lactate and malate dehydrogenase genes $(\Delta l d h$ $\Delta m d h$, hereafter LM mutant) abolished lactate production, accompanied with carbon flux redistribution (Li et al., 2012). However, no knock-out mutants of acetate producing genes, phosphotransacetylase (pta) and acetate kinase (ack), were isolated to abolish acetate formation, suggesting that these two genes are essential in C. cellulolyticum under the condition tested (Li et al., 2012). The difficulty hampered combined metabolic engineering to maximize the elimination of less useful products (acetate and lactate) as demonstrated in the triple mutant of T. saccharolyticum ( $\Delta$ ldh $\Delta$ pta $\Delta a c k$ ) (Shaw et al., 2008) and the quintuple mutant of C. thermocellum ( $\Delta h p t$, $\Delta l d h, \Delta h y d G, \Delta p f l$, and $\Delta p t a-a c k$ ) (Papanek et al., 2015). With the aim of reducing acetate formation by manipulating these essential metabolic genes, both the traditional double-crossover recombination (Heap et al., 2012) and the newly developed Cas9 nickase-triggered homologous recombination, which has been proven in C. cellulolyticum (Xu et al., 2015), were employed to deliver the cassettes of antisense RNA expressing modules into a targeted genomic locus. The RNA repression effect in plasmid transformants and chromosomal integrants was determined and compared. Then, we improved the repression effect in chromosomal integrants by using a synthetic tandem promoter. The genetic regulatory strategies established in this study will greatly expand our ability to stably tune the expression of genes for genetic and metabolic engineering of bacteria.

\section{MATERIALS AND METHODS}

\section{Plasmid Construction}

To construct plasmids expressing asRNAs, a partial transcriptional region of either the pta or ack gene, spanning from the predicted transcriptional start site to the downstream site approximately 120 bp away from the start codon, was amplified with specific primer sets (Supplementary Table S1). The exact size of asRNAs varied depending on primers used to generate PCR amplicon with the size of $>100$ bp which can trigger efficient RNA repression in bacteria (Perret et al., 2004; Wang and Kuramitsu, 2005). Then, qualified PCR products were fused with the Clostridium pasteurianum ferredoxin promoter in an inverted orientation by assembling with BamHI-linearized pRNAi control plasmid (Gibson assembly kit, NEB), generating pRNAi-pta and pRNAi-ack harboring Fd::pta asRNA module and Fd::ack asRNA module respectively.

To conduct chromosomal integration of asRNA modules via double-crossover recombination (Heap et al., 2012), plasmids containing these asRNA modules flanked by homologous arms were constructed as follows. First, DNA fragments of interest were amplified and purified separately: promoterless $m l s R$ gene amplified from pLyc1217Er (Li et al., 2012); asRNA cassettes from pRNAi and pRNAi-pta; upper and lower homologous arms from the wildtype (WT) genome; and linear backbone from pRNAi. These fragments were then mixed and assembled together using a Gibson assembly kit and the resulting reaction product was transformed into Escherichia coli for colony screening. Consequently, plasmids pLyc045 and pLyc046 were constructed with 3198up-mlsR-empty asRNA-3198down and 3198up-mlsRpta asRNA-3198down for the integration of Fd::empty and Fd::pta asRNA cassette at the selected locus. Similarly, to integrate 
the Fd::afp cassette there, pLyc048 was constructed with 3198 upmlsR-Fd::afp-3198down.

To increase asRNA expression, a tandem promoter cluster consisting of three $\mathrm{P} 4$ promoters was synthesized and then fused with the same asRNA region by overlapping PCR, generating a 3P4::pta asRNA cassette. Since Cas9 nickase-based chromosomal integration is simpler and much more efficient (Xu et al., 2015), it was applied to deliver 3P4::pta asRNA into the genome. The 23-bp target site (5'-AAGTAAGAAACATTTGGTTCCGG-3') was located in the downstream intergenic region of Ccel_3198. pCas9n-3198D with a customized donor was constructed in two steps. First, pCas9n-3198D reported previously was linearized by BamHI (Xu et al., 2015) and then assembled with both left and right homologous arms amplified from the WT genome, generating pCas9n-3198D with NcoI-containing donor. Second, the resulting plasmid was linearized by NcoI for the assembly with the 3P4::pta asRNA cassette, generating pCas9n-3198Ddonor. Descriptions of all plasmids used in this study were listed in Table 1.

\section{Bacterial Strains and Culture Conditions}

Escherichia coli Top10 (Invitrogen) was used for molecular cloning. Transformants were grown at $37^{\circ} \mathrm{C}$ in LuriaBertani medium supplemented with kanamycin $(50 \mu \mathrm{g} / \mathrm{ml})$ or chloramphenicol $(15 \mu \mathrm{g} / \mathrm{ml})$ when required. Clostridium cellulolyticum $\mathrm{H} 10$ and developed strains were cultured anaerobically at $34^{\circ} \mathrm{C}$ in VM media supplemented with $2.0 \mathrm{~g} / \mathrm{L}$ yeast extract and various carbon sources (Higashide et al.,
2011). Transformants of $\mathrm{H} 10$ and $\mathrm{LM}$ mutant were selected by erythromycin $(15 \mu \mathrm{g} / \mathrm{ml})$ or thiamphenicol $(15 \mu \mathrm{g} / \mathrm{ml})$. Colonies of C. cellulolyticum strains were developed on solid VM plates containing $1 \%(\mathrm{w} / \mathrm{v})$ agar, $5 \mathrm{~g} / \mathrm{L}$ cellobiose and antibiotics. Plasmid transformants were generated by transforming the corresponding plasmids. Chromosomal integrants, WT-G and WT-G-afp, were generated by transforming WT with pLyc045 and pLyc048, respectively. Chromosomal integrants, LM-G, LM-G-PTA and LM3P, were generated by transforming the LM mutant with pLyc045, pLyc046, and pCas9n-3198D-donor, respectively. All constructed strains are listed in Table $\mathbf{1 .}$

\section{C. cellulolyticum Transformation}

Clostridium cellulolyticum electro-competent cells and methylated plasmids were prepared as previously described (Li et al., 2014). Briefly, C. cellulolyticum strains were grown at $34^{\circ} \mathrm{C}$ in liquid $\mathrm{VM}$ medium with $5 \mathrm{~g} / \mathrm{L}$ cellobiose and $2 \mathrm{~g} / \mathrm{L}$ yeast extract until reaching an $\mathrm{OD}_{600}=0.3-0.5$. The cell culture was then chilled on ice and then centrifuged at $4^{\circ} \mathrm{C}$ and $3,000 \mathrm{~g}$ for $8 \mathrm{~min}$, and the cell pellets were washed at least three times with an equal volume of ice-cold anoxic electroporation buffer $\left(270 \mathrm{mM}\right.$ sucrose, $1 \mathrm{mM} \mathrm{MgCl}_{2}$ and $5 \mathrm{mM}$ sodium phosphate buffer, $\mathrm{pH}$ 7.4). Lastly, competent cells made from every $10 \mathrm{ml}$ of cell culture were resuspended in $100 \mu \mathrm{l}$ chilled electroporation buffer for further use. Plasmid DNA was methylated with MspI methyltransferase (NEB), followed by DNA purification and quantification. For each transformation, a $100 \mu \mathrm{l}$ cell suspension was mixed

TABLE 1 | Plasmids and strains used in this study.

\begin{tabular}{|c|c|c|}
\hline Name & Description & Reference \\
\hline \multicolumn{3}{|l|}{ Strain } \\
\hline E. coli TOP10 & Host cells for plasmid construction & Invitrogen \\
\hline WT & Wildtype C. cellulolyticum $\mathrm{H} 10$ & ATCC \\
\hline WT-P & WT with pRNAi control plasmids & This study \\
\hline WT-P-pta & WT with pRNAi-pta plasmids & This study \\
\hline WT-P-ack & WT with pRNAi-ack plasmids & This study \\
\hline WT-P-afp & WT with pFd-AFP plasmids & (Xu et al., 2015) \\
\hline WT-G & WT with a chromosomal Fd::empty cassette & This study \\
\hline WT-G-afp & WT with a chromosomal Fd::afp cassette & This study \\
\hline LM & $\Delta / d h \Delta m d h$ & (Li et al., 2012) \\
\hline LM-P & LM with pRNAi plasmids & This study \\
\hline LM-P-pta & LM with pRNAi-pta plasmids & This study \\
\hline LM-G & LM with a chromosomal RNAi control & This study \\
\hline LM-G-pta & LM with a chromosomal Fd::pta asRNA cassette & This study \\
\hline \multicolumn{3}{|l|}{ Plasmid } \\
\hline pRNAi & $\mathrm{CMP}^{r}$ in E. coli; $\mathrm{TMP}^{r}$ in H10; Fd::empty cassette & (Xu et al., 2015) \\
\hline pRNAi-pta & pRNAi derivative with a Fd::pta asRNA cassette & This study \\
\hline pRNAi-ack & pRNAi derivative with a Fd::ack asRNA cassette & This study \\
\hline pLyc045 & pRNAi derivative with 3198up-mlsR-Fd::empty-3198down & This study \\
\hline pLyc046 & pRNAi derivative with 3198up-mlsR-Fd::pta asRNA-3198down & This study \\
\hline pFd-AFP & pRNAi derivative with a Fd::afp cassette & (Xu et al., 2015) \\
\hline pLyc048 & pRNAi derivative with 3198up-mlsR-Fd::afp-3198down & This study \\
\hline pCas9n-3198D & pRNAi derivative with a cas9 nickase and a gRNA targeting the 3198D site & (Xu et al., 2015) \\
\hline pCas9n-3198D with donor & pCas9n-3198D derivative with left arm-3P4::pta asRNA-right arm & This study \\
\hline
\end{tabular}


with $2.0 \mu \mathrm{g}$ of methylated plasmids and then electroporated in a $2-\mathrm{mm}$ cuvette $(1.25 \mathrm{kV}, 5 \mathrm{~ms}, 1$ square pulse) with a Gene Pulser Xcell (Bio-Rad) in the anaerobic chamber. After electroporation, cells were recovered for $12-24 \mathrm{~h}$ in antibioticfree VM medium with $5 \mathrm{~g} / \mathrm{L}$ cellobiose and $2 \mathrm{~g} / \mathrm{L}$ yeast extract, and then selected by appropriate antibiotics on agar VM plates.

\section{Enzyme Activity Assay}

To measure enzyme activities, cell-free extracts were made from cellobiose-grown C. cellulolyticum strains at the mid-log phase using CelLytic B cell lysis reagent (Sigma). Crude extracts were centrifuged at $14,000 \mathrm{~g}$ at $4^{\circ} \mathrm{C}$ for $10 \mathrm{~min}$ to remove insoluble cell debris. Then, the protein concentration was determined with a BCA assay kit (Thermo Scientific), using bovine serum albumin as a standard. Crude protein samples were stored on ice until assayed. One unit of activity is defined as the amount of enzyme that catalyzes the conversion of one micromole substrate per minute under the experimental conditions. The specific activity was defined as the units of enzyme activity per $\mathrm{mg}$ of total protein.

Acetate kinase activity was measured in the direction of acyl phosphate formation (Rose, 1955). The reaction was initiated by adding $0.4 \mu \mathrm{g}$ of protein sample to $320 \mu \mathrm{l}$ reaction mixture [200 mM Tris- $\mathrm{HCl}$ (pH 7.4), $10 \mathrm{mM}$ ATP, $10 \mathrm{mM} \mathrm{MgCl2}$, $6 \%(w / v)$ hydroxylamine hydrochloride (neutralized with $\mathrm{KOH}$ before addition), and $267 \mathrm{mM}$ potassium acetate]. The reaction was incubated at $25^{\circ} \mathrm{C}$ for $10 \mathrm{~min}$ and stopped by adding $320 \mu \mathrm{l}$ of $10 \%(\mathrm{w} / \mathrm{v})$ ice-cold trichloroacetic acid. The experimental control was made with boiled protein samples in the above reaction mixture. Color was developed by adding $320 \mu 12.5 \%$ (w/v) $\mathrm{FeCl} 3$ in $2.0 \mathrm{~N} \mathrm{HCl}$. The absorbance at $540 \mathrm{~nm}$ was measured with a Biowave II spectrophotometer (WPA). An extinction coefficient of $0.169 / \mathrm{mM} / \mathrm{cm}$ was used to calculate the activity of acetate kinase.

Phosphotransacetylase activity was measured by monitoring the liberation of coenzyme A at $405 \mathrm{~nm}$ (Andersch et al., 1983). The reaction was initiated by adding $2 \mu \mathrm{g}$ of cellfree extracts to $1 \mathrm{~mL}$ of reaction mixture $[0.1 \mathrm{M}$ potassium phosphate buffer ( $\mathrm{pH}$ 7.4), $0.2 \mathrm{mM}$ acetyl-CoA, $0.08 \mathrm{mM}$ $5,5^{\prime}$-dithio-bis (2-nitrobenzoate)] and then incubated at $25^{\circ} \mathrm{C}$ for $10 \mathrm{~min}$. The experimental control was made with boiled protein samples in the above reaction mixture. The absorbance at $405 \mathrm{~nm}$ was measured with a Biowave II spectrophotometer (WPA). An extinction coefficient of $13.6 / \mathrm{mM} / \mathrm{cm}$ was used to calculate phosphotransacetylase activity.

Aldehyde dehydrogenase activity was measured by monitoring NADH oxidation which decreases absorbance at $340 \mathrm{~nm}$ (Brown et al., 2011). Protein samples $(10 \mu \mathrm{l})$ were added to $1 \mathrm{~mL}$ reaction mixture $[100 \mathrm{mM}$ Tris- $\mathrm{HCl}$ (pH 7.6), $1 \mathrm{mM}$ DTT buffer, $5 \mu \mathrm{M} \mathrm{FeSO}_{4}, 0.5 \mathrm{mM} \mathrm{NADH}$, $55 \mathrm{mM}$ acetaldehyde] and incubated at $34^{\circ} \mathrm{C}$ for $20 \mathrm{~min}$ before absorbance measurement. The experimental control was made with boiled protein samples in the above reaction mixture. An extinction coefficient of $6.22 / \mathrm{mM} / \mathrm{cm}$ was used to calculate aldehyde dehydrogenase activity.

\section{Measurement of Cell Growth and Fermentation Products}

Clostridium cellulolyticum strains were revived in VM media with $5 \mathrm{~g} / \mathrm{L}$ cellobiose, and antibiotic was added if necessary. The cellobiose-grown cultures at an $\mathrm{OD}_{600}$ of $0.5-0.7$ were used for $1 \%$ inoculation into $50 \mathrm{ml}$ fresh VM media with $5 \mathrm{~g} / \mathrm{L}$ cellobiose, $10 \mathrm{~g} / \mathrm{L}$ Avicel PH101 crystalline cellulose (Sigma) or $10 \mathrm{~g} / \mathrm{L}$ beach wood xylan (Sigma). Each strain had three biological replicates. Cell growth on cellobiose was profiled by monitoring OD600 with a spectrophotometer. When grown on cellulose and xylan, $1 \mathrm{~mL}$ of cell culture was sampled and then stored at $-80^{\circ} \mathrm{C}$ for metabolite measurements.

Cell biomass on cellulose was estimated by total protein measurement. The cells were lysed with $0.2 \mathrm{~N} \mathrm{NaOH} / 1 \% \mathrm{w} / \mathrm{v}$ SDS solution for $60 \mathrm{~min}$ at $25^{\circ} \mathrm{C}$, and then neutralized with $0.8 \mathrm{~N} \mathrm{HCl}$. After centrifugation at $12,000 \mathrm{~g}$ for $10 \mathrm{~min}$, the supernatant was used for protein quantification with a BCA assay kit.

To measure fermentation products (including lactate, acetate, and ethanol), the fermentation broth was filtered through $0.2 \mu \mathrm{m}$ filters, acidified with $0.025 \% \mathrm{H}_{2} \mathrm{SO}_{4}$ and then subjected to high-performance liquid chromatography (HPLC) analyses with an Agilent 1200 system (Agilent Technologies) equipped with a variable-wavelength $(190-600 \mathrm{~nm}$ ) detector (with UV absorption measured at $245 \mathrm{~nm}$ ) and an ion-exclusion column (Aminex HPX-87H; $300 \mathrm{~mm} \times 7.8 \mathrm{~mm}$; Bio-Rad Laboratories, Hercules, CA, United States). HPLC operating parameters included a column temperature at $65^{\circ} \mathrm{C}, 0.025 \%$ sulfuric acid as the mobile phase at a flow rate of $0.6 \mathrm{ml} / \mathrm{min}$ and $50 \mu \mathrm{l}$ sample injected (Hemme et al., 2011). Referring to the corresponding standard curves, the concentration of each product was calculated.

\section{Quantitative Real-Time PCR}

To compare the gene copy number and the transcript amount of afp gene in P-AFP transformant and G-AFP integrant, qRTPCR was conducted as follows. Cell samples were collected from cellobiose-grown cultures at mid-log phase $\left(\mathrm{OD}_{600}=\sim 0.45\right)$. To compare gene copy number, DNA was extracted by heating at $98^{\circ} \mathrm{C}$ for $6 \mathrm{~min}$. Heat-treated samples were centrifuged to remove insoluble cell debris. Then, the supernatants were subjected to qRT-PCR analysis using iTaq SYBR Green Supermix with ROX (Bio-Rad) on a Bio-Rad iQ5 thermal cycler. The recA gene in the genome was used as an internal calibrator to determine the copy number of afp gene. Primers used in qRT-PCR are listed (Supplementary Table S1). Results were analyzed with the Pfaffl method (Pfaffl, 2001).

To compare the transcript amount of afp gene by qRT-PCR, cells were lysed by TRIzol Reagent (Invitrogen) followed by total RNA extraction and purification with NucleoSpin RNAII kit (Macherey-Nagel). SuperScript III Reverse Transcriptase (Invitrogen) was applied to convert RNA to cDNA by following the manufacturer's protocol. cDNA products were diluted as appropriate and used as templates for qRT-PCR. Similarly, results were analyzed with the Pfaffl method using $r e c A$ as the reference gene (Pfaffl, 2001). 


\section{Microscopy and Flow Cytometry}

Fluorescence intensity of the anaerobic fluorescence protein was evaluated by fluorescent microscopy and flow cytometry. C. cellulolyticum strains at the mid-log phase were harvested, washed twice with the anaerobic PBS buffer and then suspended in the same buffer before loading onto microscope slides. Slides were imaged using Olympus BX51 fluorescence microscope equipped with optical filter sets with excitation at $490 \mathrm{~nm}$ and emission at $525 \mathrm{~nm}$ for the green fluorescence. The images were collected by an Olympus DP71 digital camera.

Flow cytometry analysis was performed on a BD Accuri C6 flow cytometer (BD Biosciences) (Li et al., 2014). All samples were diluted with the anaerobic PBS buffer to similar concentrations, then run through the flow cytometer under aerobic condition following the manufacturer's instructions. The run limit was set up as 10,000 events at a slow flow rate, the threshold as 40,000 on FSC-H. The fluorescence was detected with a FL1 detector with a 530/30 filter. The data were collected and analyzed with the CFlow software.

\section{RESULTS AND DISCUSSION}

\section{Plasmid-Based Antisense RNA Expression}

To test the use of asRNA molecules to repress acetate production in C. cellulolyticum, we targeted pta encoding phosphotransacetylase (PTA) and ack encoding acetate kinase (ACK), both of which are key to produce acetate from acetylCoA (Figure 1A) and essential for cell survival (Li et al., 2012). For each target gene, its $5^{\prime}$ transcriptional region with a length of approximately $120 \mathrm{bp}$ was inserted in a reverse orientation under the control of a ferredoxin promoter to produce asRNAs which will interfere with the stability and translation of target transcripts (Figure 1B) (Thomason and Storz, 2010). The empty asRNA plasmid (pRNAi), customized pRNAi-pta and pRNAi-ack plasmids targeting $p t a$ and $a c k$, respectively, were constructed and transformed into WT, generating WT-P control, WT-Ppta and WT-P-ack transformants (where P means plasmidbased expression). Then, we examined the repression effect of designed asRNAs by measuring enzyme activities of PTA and ACK in these strains that were grown on $5 \mathrm{~g} / \mathrm{L}$ cellobiose (Figure 1C). Our results showed that (i) PTA activity in WT-Ppta $(0.54 \pm 0.01 \mathrm{U} / \mathrm{mg})$ was decreased to $65 \%$ of WT-P control $(0.83 \pm 0.02 \mathrm{U} / \mathrm{mg})$ and WT-P-ack $(0.84 \pm 0.02 \mathrm{U} / \mathrm{mg})$; (ii) ACK activity was barely changed in WT-P-ack $(9.51 \pm 0.21 \mathrm{U} / \mathrm{mg})$ compared to WT-P $(9.19 \pm 0.92 \mathrm{U} / \mathrm{mg})$ and WT-P-pta $(8.69 \pm 0.36 \mathrm{U} / \mathrm{mg})$. The $p t a$ asRNAs performed better than ack asRNAs in repression. The strain expressing pta asRNAs was further characterized.

There are a few possible reasons that could explain the observed difference in repression exerted by pta and ack asRNAs. AsRNA repression follows a threshold linear response (Georg and Hess, 2011), which suggests that RNA repression


FIGURE 1 | (A) Major metabolic pathways in C. cellulolyticum. Acetyl-CoA as a key intermediate metabolite, apart from being used to produce ethanol, can be converted to acetyl-phosphate by phosphotransacetylase (PTA, encoded by pta gene) and then to acetate by acetate kinase (ACK, encoded by ack gene). L-lactate dehydrogenase $(\mathrm{LDH})$ and $\mathrm{L}$-malate dehydrogenase $(\mathrm{MDH})$ are functional in one-step lactate production from pyruvate. Dashed arrows refer to multiple enzymatic reactions. (B) Design of antisense RNAs (asRNAs) to repress pta and ack genes. For each target gene, the transcriptional region spanning from the predicted transcriptional start site (TSS) to the downstream site approximately 120-bp from the start codon (ATG), containing the Shine-Dalgarno sequence (SD), was amplified and reversely inserted downstream of the ferredoxin (Fd) promoter, generating the Fd::asRNA module. AsRNAs would interfere with the transcription, stability and translation of the target gene. (C) Enzyme assays of PTA and ACK in crude cell-free extracts. Mean and standard deviations of specific enzyme activities were calculated from three biological replicates. 
only occurs when the abundance of asRNAs is higher than a certain threshold and then with a continuing increase in asRNA abundance, repression will gradually increase. It is possible that WT-P-pta and WT-P-ack strains have different thresholds depending on the abundance of pta or ack transcripts, and the abundance of asRNAs may vary due to differential vulnerability to ribonucleases. In addition, the RNA structure is important to the physical binding between asRNAs and target transcripts that is necessary for RNA repression. RNA structure prediction (Gruber et al., 2008) found that the ack target region is more likely to form a secondary structure (Supplementary Figure S1), which may influence asRNA binding. Although we did not evaluate the extent that these factors could affect RNA repression, our study indicated the variability of asRNA repression and suggested the potential importance of asRNA design and promoter activity in maximizing RNA repression.

\section{Metabolic Changes in Knock-Down Strains}

We measured the titers of three major metabolites (lactate, acetate, and ethanol) at the end of batch fermentations to determine if acetate production was decreased. With $10 \mathrm{~g} / \mathrm{L}$ cellulose, the WT-P-pta strain produced lactate, acetate, and ethanol in a molar ratio of $0.93: 1.37: 1$, compared to $1.75: 1.49: 1$ in WT-P control (Supplementary Table S2). The acetate titer in WT-P-pta was decreased about $15 \%$ relative to the titer of WT-P (Figure 2). Interestingly, the lactate titer was decreased

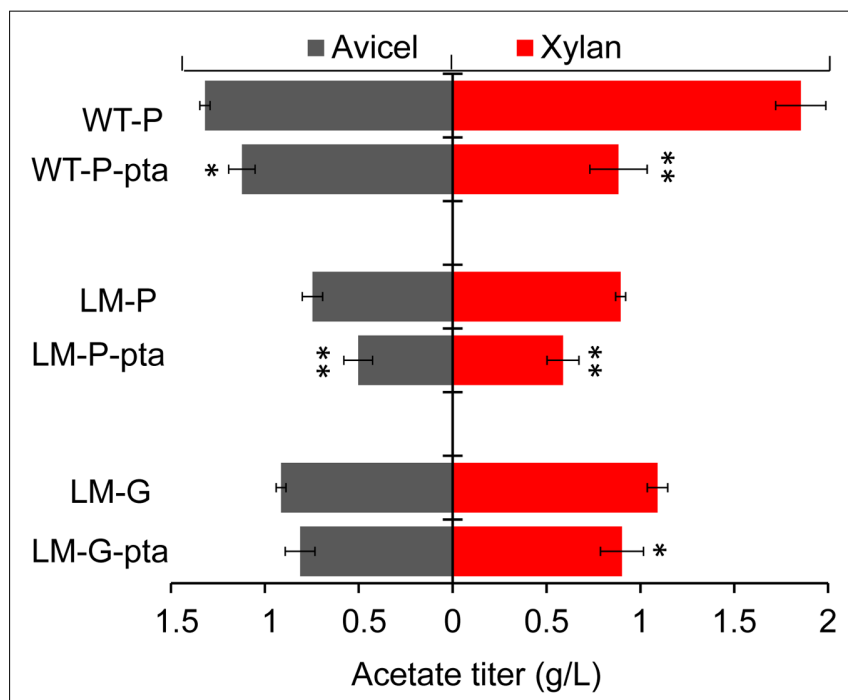

FIGURE 2 | Comparison of acetate titers produced on $10 \mathrm{~g} / \mathrm{L}$ Avicel cellulose (Left) and $10 \mathrm{~g} / \mathrm{L}$ xylan (Right). Strain names are labeled on the left, where WT-P and LM-P represent empty vector transformants of wildtype and lactate production-deficient mutant LM respectively, LM-G represents the chromosomal integrant with an empty repression module, all others carry a pta repression module either in plasmid (WT-P-pta and LM-P-pta) or chromosome (LM-G-pta). Error bar represents the standard deviation of three biological replicates. The asterisk $\left({ }^{*}\right)$ indicates statistically significant differences between the engineered strain and its corresponding control (student's $t$-test, ${ }^{*} P<0.05,{ }^{* *} P<0.01$ ). more than $50 \%$ in WT-P-pta, but ethanol production was not significantly changed (Supplementary Table S2). When both strains were grown on $10 \mathrm{~g} / \mathrm{L}$ xylan, acetate became the major product, which is consistent with previous studies (Li et al., 2012); strikingly, WT-P-pta substantially reduced acetate titer to less than $48 \%$ of WT-P (Figure 2), corresponding to a molar ratio of acetate to ethanol of 4.11:1 in WT-P-pta versus 6:1 in WT$\mathrm{P}$ (Supplementary Table S2). Notably, the pta asRNAs expressed in WT performed very well in reducing acetate production even though carbon sources greatly change metabolic profiles. The unexpected decrease in lactate titer on cellulose, as a side effect of manipulating acetate-producing genes, suggests a more sophisticated metabolic regulatory network in this strain, which was also supported by the reported decrease in acetate production in the lactate production-deficient LM mutant ( $\mathrm{Li}$ et al., 2012). However, in C. thermocellum the $\Delta$ pta knockout mutant dramatically increased lactate titer (Argyros et al., 2011), which is in contrast to the accompanying decrease in lactate titer in the pta knockdown mutant of C. cellulolyticum. It seems like Clostridium strains employ different strategies to coordinate metabolic networks. In addition, despite the operability of pta disruption in some strains, the resulting effectiveness in acetate formation varies a lot. For example, pta deletion reduced acetate by just $14 \%$ in Clostridium tyrobutyricum (Zhu et al., 2005), but completely eliminated it in C. thermocellum (Argyros et al., 2011) and Thermoanaerobacterium saccharolyticum (Shaw et al., 2008).

Next, we transformed pRNAi-pta into the lactate productiondeficient LM mutant to generate an LM-P-pta strain that should be deficient in both lactate and acetate production. A control strain, LM-P, was created in parallel by transforming pRNAi that cannot express any specific asRNAs. Metabolic profiling revealed that on $10 \mathrm{~g} / \mathrm{L}$ cellulose, the LM-P control produced lactate, acetate and ethanol with a molar ratio of 0.04:0.55:1 (Supplementary Table S2); LM-P-pta made negligible lactate, a $33 \%$ decrease in acetate titer (Figure 2) and an $86 \%$ increase in ethanol titer, resulting in a molar ratio of 0.001:0.20:1 (lactate: acetate: ethanol). On $10 \mathrm{~g} / \mathrm{L}$ xylan, the titers of lactate and acetate in LM-P-pta were decreased about 82 and 34\% (Figure 2), respectively, and ethanol titer was slightly increased, corresponding to a molar ratio of 0.06:0.83:1 in LM-P and 0.01:0.51:1 in LM-P-pta (Supplementary Table S2). Hence, with the customized pta asRNAs expressed in transformants, we successfully manipulated both lactate and acetate producing pathways simultaneously.

Comparing the molar ratio of the three major end products (lactate, acetate and ethanol) in the control and asRNA expressing strains, we found that both WT-P-pta and LM-P-pta produced an equal molar amount of ethanol by generating less lactate and acetate, regardless of carbon source (Supplementary Table S2). In another word, these repression strains recovered more carbon in the form of ethanol. For instance, when LM-P-pta was grown on cellulose, $83 \%$ of the carbons used to produce the three major metabolites were accounted for in the ethanol, $21 \%$ higher than the corresponding control (Supplementary Table S2). This demonstrates a successful manipulation of essential metabolic genes to divert carbon flux toward ethanol production. 


\section{Chromosomal Integration and Functional Analyses}

In light of the effectiveness of pta asRNAs in reducing acetate production, we attempted to integrate the asRNA-expressing module into the genome of the LM mutant in such a way that resulting integrants can work stably and desirably without using antibiotics. To do so, step-wise double-crossover recombination was initially applied before the use of Cas9 technology in Clostridia (Heap et al., 2012) (Figure 3A). The integration site was immediately downstream of the sole bifunctional acetaldehyde-CoA/alcohol dehydrogenase-encoding gene ( $a d h E)$ in C. cellulolyticum (Ccel_3198). The specific integration can generate an artificial bicistronic operon containing the open reading frames of adhE and $m l s R$ under the control of the native adhE promoter, consequently enabling counter selection of double-crossover events with erythromycin. During the screening of double-crossover events, pure LM-G-pta integrants (where G indicates genome/chromosome-based expression) were isolated after two rounds of plate streaking. Targeted integration in LM-G-pta and LM-G controls was verified by PCR amplification (Figure 3B) and amplicon sequencing.

The functionality of the integrated P4::pta asRNA module was evaluated by measuring PTA activity and fermentation products. In comparison, the crude extract of cellobiose-grown LM-G-pta integrant presented a lower PTA activity $(0.92 \pm 0.04 \mathrm{U} / \mathrm{mg})$ that was $89 \%$ of $\mathrm{LM}-\mathrm{G}$ control $(1.13 \pm 0.06 \mathrm{U} / \mathrm{mg})$, indicating the integrated module was still functional but did not perform as well as the plasmid-based repression in WT-P-pta (Figure 1C). Metabolically, the acetate titer in LM-G-pta did not significantly reduce on $10 \mathrm{~g} / \mathrm{L}$ cellulose but dropped $17 \%$ on $10 \mathrm{~g} / \mathrm{L}$ xylan (Figure 2). The overall molar ratios (lactate: acetate: ethanol) were changed from $0.05: 0.59: 1$ in LM-G to $0.01: 0.31: 1$ in
A

\section{Chromosome}
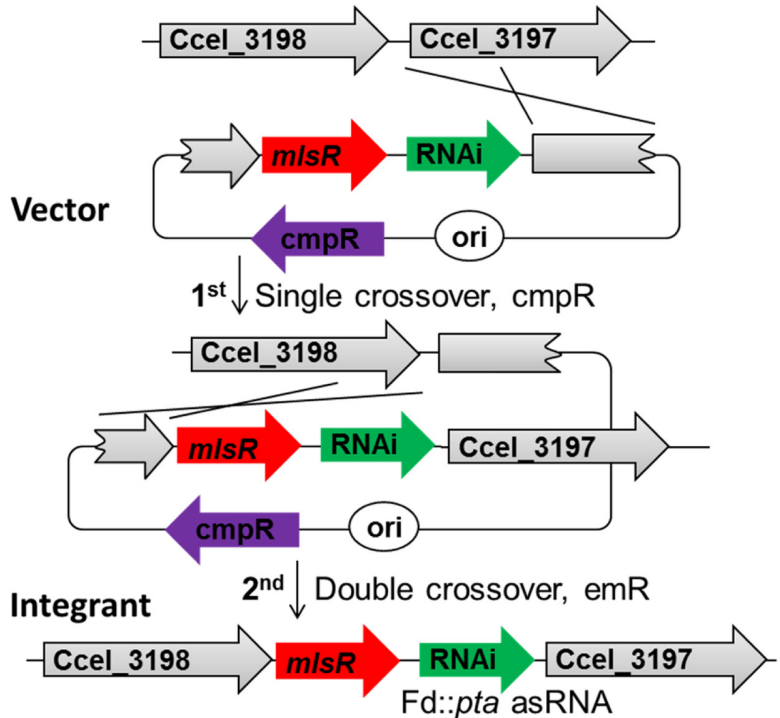

B

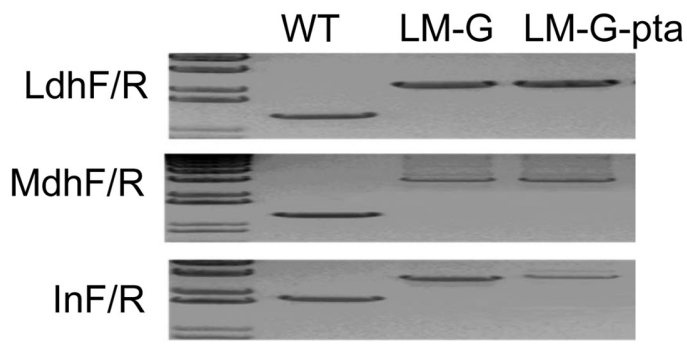

C

\section{Chromosome}

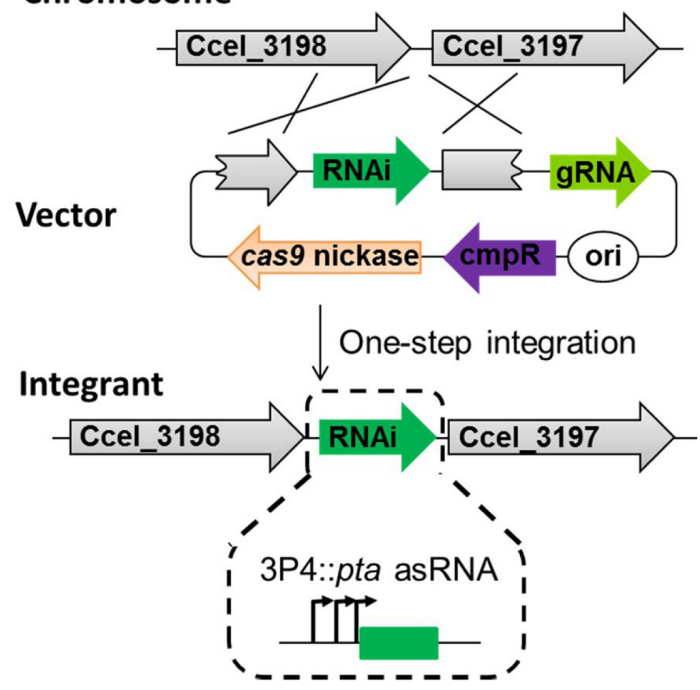

D

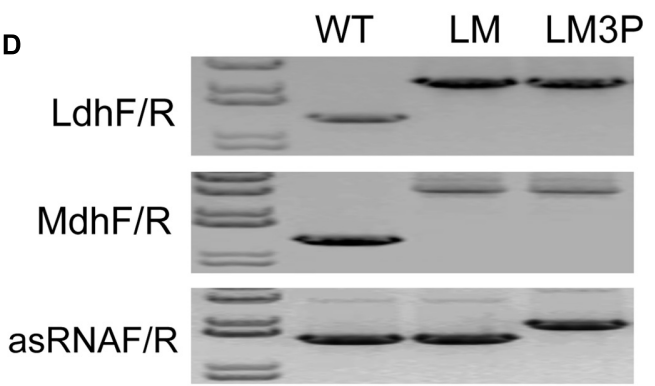

FIGURE 3 | Chromosomal integration of functional modules via double-crossover recombination (A,B) and the Cas9 nickase genome editing tool (C,D). The integration site was located in the intergenic region between Ccel_3198 and Ccel_3197. (A) Generation of stable double-crossover clones, LM-G and LM-G-pta, using pLyc045 and pLyc046, respectively. The first step was to screen thiamphenicol-resistant single-crossover clones generated by plasmid integration. The second step was to select erythromycin-resistant double-crossover clones as a result of plasmid excision. Finally, modified genomic loci in candidate clones were verified by PCR with specific primers, LdhF/R for $\Delta / d h$ identification, MdhF/R for $\Delta$ mdh identification and InF/R for module integration (B). (C) Generation of stable chromosomal integrants, LM3P and LM3PS, by the Cas9 nickase genome editing tool. By transforming pCas9n-3198D-donor into the LM mutant, integrants were generated within a single step. (D) Modified genome loci in all integrants were then verified by PCR with specific primers, asRNAF/R for RNAi module integration. LM is a double mutant $(\Delta / d h \Delta m d h)$; LM-G-pta and LM3P are triple mutants ( $\Delta / d h \Delta m d h \Delta p t a)$. 
LM-G-pta when cultured on cellulose, and correspondingly from 0.09:1.13:1 to 0.04:0.84:1 on xylan (Supplementary Table S2). In general, the integrant was not comparable with the aforementioned transformant in repressing enzymatic and metabolic activities. Previous studies have found that small RNA repression follows a threshold-linear model distinct from protein-mediated repression (Levine et al., 2008; Georg and Hess, 2011). In this case, with a fixed transcription rate of chromosomal pta gene, switching from plasmid-based to chromosome-based asRNA expression presumably reduces asRNA dosage, which would weaken the repression of acetate formation.

\section{Evaluation of the Gene-Dosage Effect between Transformants and Chromosomal Integrants}

To determine if chromosomal integration mitigates gene activity and how strong the effect is, an afp gene encoding anaerobic fluorescent protein was introduced into either the plasmid (P-AFP) or the genome (G-AFP) and then their respective activities were visualized and compared. As expected, P-AFP presented much stronger signal intensity than G-AFP under fluorescent microscopy (Figure 4A). Then, quantification of the fluorescence signal by flow cytometry revealed that the signal intensity of P-AFP was 1.73-fold higher than that of G-AFP (Figure 4B); when compared to corresponding negative controls (P-CK and G-CK), P-AFP and G-AFP generated 2.79-fold and 1.65 -fold greater fluorescent intensity, respectively. The lower signal intensity of G-AFP directly reflects a lower AFP activity and presumably indicates a lower amount of afp transcripts produced in G-AFP. Quantitative real-time PCR (qRT-PCR) analysis supported this assumption, showing that relative to G-AFP, P-AFP harbored a 12-fold higher abundance in afp gene copy number (Figure 4C) and a 36-fold higher abundance in afp transcript (Figure 4D). These results together indicate that chromosomal integration substantially altered the dosage of gene expression and then diminished gene activity. High-copy number pJIR750 derivatives, including pLyc17 used here to generate P-AFP transformants, have also been reported in Clostridium perfringens, which carried as many as 18 copies (Cheung et al., 2009).

\section{Improved Repression of Acetic Acid Production by a Tandem Repetitive Promoter}

To overcome the weakened asRNA repression observed with chromosomal integration, we attempted to improve promoter activity to increase asRNA production by introducing a tandem promoter which consists of three $\mathrm{P} 4$ repeats, named $3 \mathrm{P} 4 . \mathrm{P} 4$ is a 36-bp synthetic promoter with an activity comparable to the strong ferredoxin $(\mathrm{Fd})$ promoter in C. cellulolyticum (Xu et al., 2015). Then, a 3P4::pta asRNA module was constructed and integrated into the LM genome at the same locus by a Cas9 nickase-based editing tool (Xu et al., 2015) (Figure 3C), generating a LM3P integrant $(\Delta l d h \Delta m d h \Delta p t a)$. After transforming the single all-in-one vector, we randomly picked three antibiotic-resistant transformants, all of which were verified to be correct integrants by PCR amplification (Figure 3D) and amplicon sequencing. Methodologically,
A
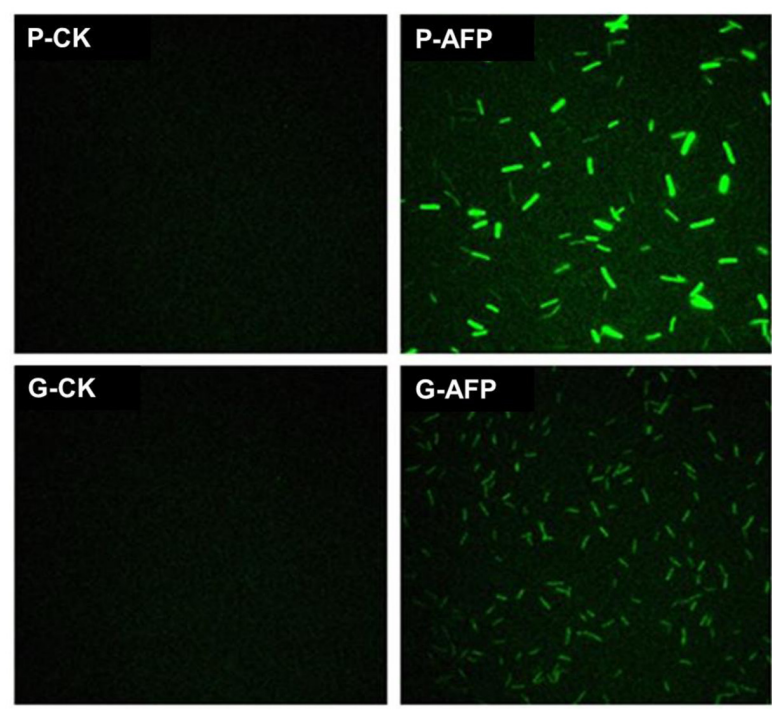

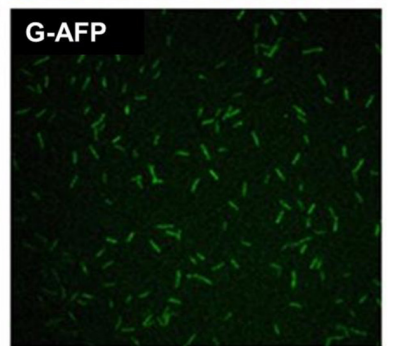

B
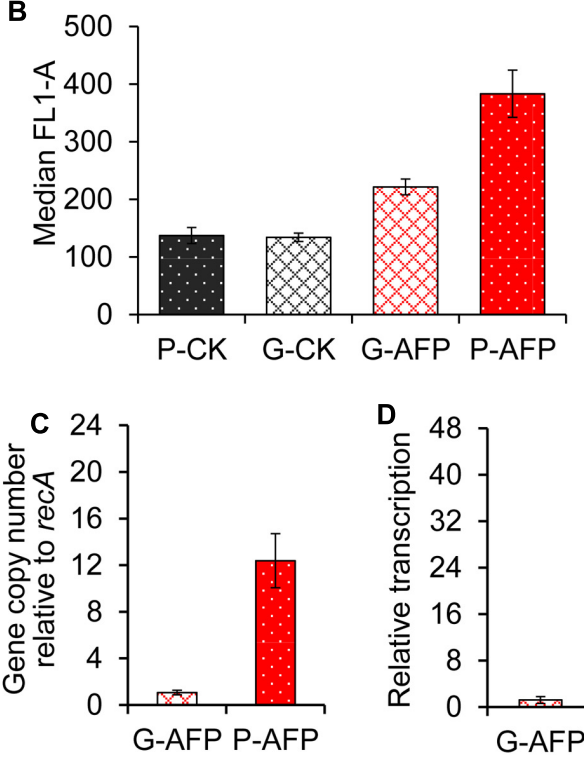

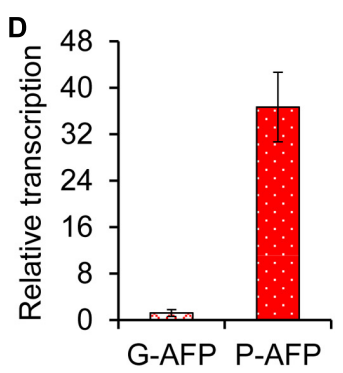

FIGURE 4 | Expression of anaerobic fluorescent protein in the P-AFP transformant and the G-AFP integrant. (A) Fluorescence microscopy of cellobiose-grown cells at the mid-log phase. P-CK and G-CK were corresponding controls of P-AFP and G-AFP that carry the reporter gene in plasmid and chromosome, respectively. (B) Quantification of fluorescent signal intensity with flow cytometry. (C) Relative afp gene copy number in both G-AFP and P-AFP by reference to the single chromosomal recA gene. (D) qRT-PCR comparison of afp transcript levels between G-AFP and P-AFP, with normalization to recA calibrator. Error bar represents the standard deviation of three biological replicates. 
although both double-crossover recombination and Cas9 nickase-triggered homologous recombination have the ability to integrate asRNA modules as shown in Figure 3, the latter presents multiple advantages, such as markerless editing, one-step generation and high editing efficiency (Xu et al., 2015).

The chromosomal asRNA module is supposed to repress the pta gene independent of plasmid-borne elements and antibiotic utilization. When grown on $5 \mathrm{~g} / \mathrm{L}$ cellobiose, both LM and LM3P presented similar biomass yields and growth rates $\left(\mu=0.13 \mathrm{~h}^{-1}\right)$ (Figure 5A), almost double the growth rate of WT $\left(\mu=0.08 \mathrm{~h}^{-1}\right)$ while LM3P's acetate titer decreased by $28 \%$ relative to LM (Figure 5B). On $10 \mathrm{~g} / \mathrm{L}$ cellulose, LM3P produced similar amount of cell biomass as LM but significantly reduced the acetate titer by $27 \%$ (Figure $5 \mathrm{C}$ ), suggesting much stronger gene repression triggered by the integrated 3P4::pta asRNA module than by the previous Fd::pta asRNA module in LM-G-pta. The enhanced repression in LM3P even got close to the plasmid-based repression in LM-P-pta. In addition, LM3P produced less ethanol than LM on cellulose (Figure $5 \mathrm{C}$ ). This reduction was not due to a negative effect of chromosomal integration on the neighboring $a d h E$ gene since the alcohol dehydrogenase activity responsible for acetaldehyde reduction was not reduced but instead increased in the crude extracts of LM3P (Supplementary Table S3). It is possible that cellular redox balancing strategies changed the reducing power for ethanol production and the carbon flow for acetate production (Desvaux et al., 2000).

Although CRSIPRi also can allow stable manipulation by integrating both non-catalytic CRSIPR endonuclease and gRNA cassettes, it is challenging to introduce the large-size endonuclease genes into microbial chromosomes (Kung et al., 2013), and whether the permanent expression of foreign CRISPRi will interfere with host CRISPR machinery or host physiology needs to be evaluated in specific hosts (Barrangou, 2015; Luo et al., 2016). Our study used transient plasmid-dependent Cas9n to deliver stand-alone asRNA modules into the genome, able to circumvent aforementioned challenges. It would also be interesting to systematically compare the repression efficiency and specificity of asRNAs and CRISPRi in bacterial hosts.

\section{CONCLUSION}

Antisense RNA-mediated repression worked well in both the C. cellulolyticum wildtype and LM mutant to repress pta
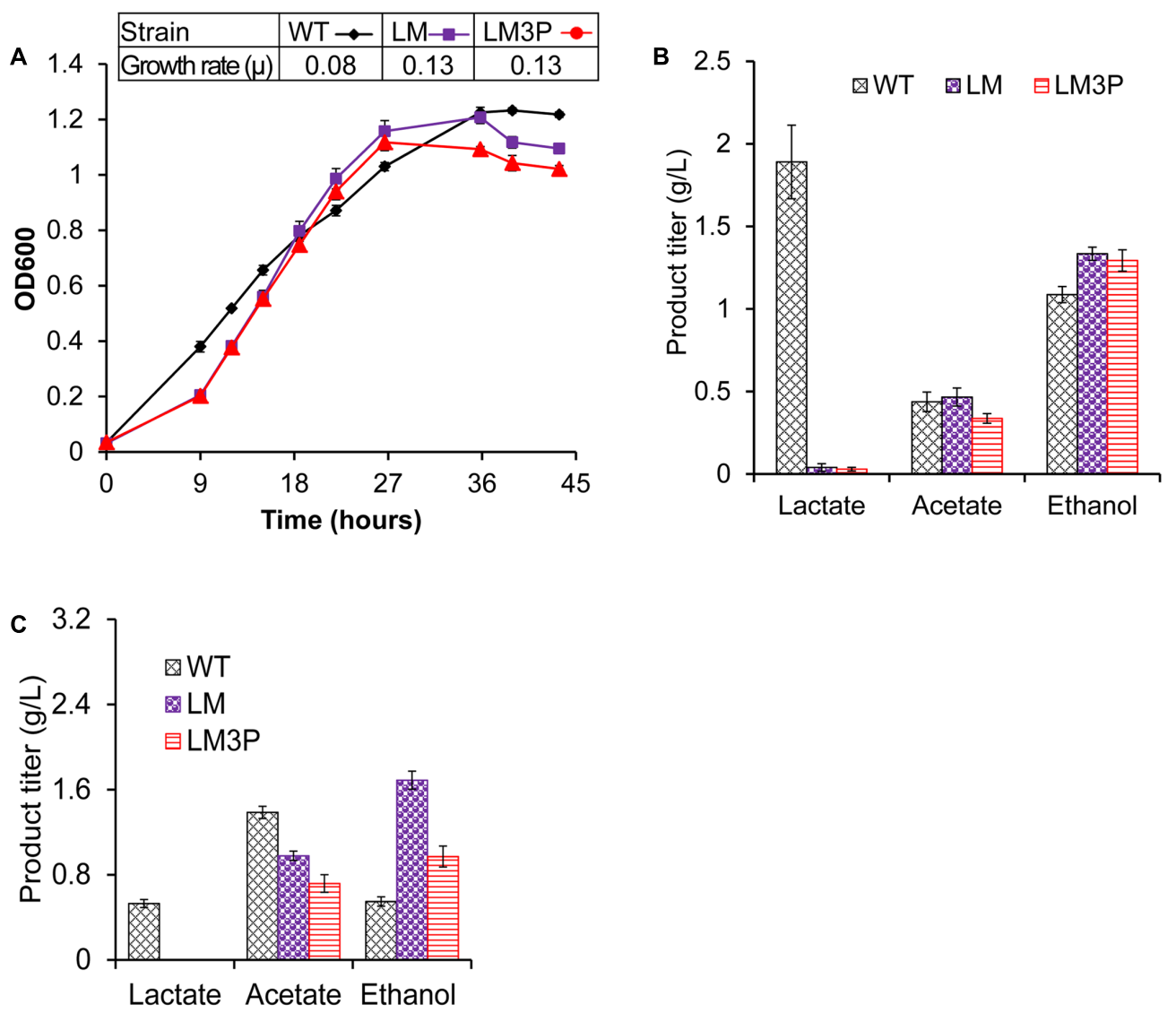

FIGURE 5 | Comparison of growth profiling and product measurement between wildtype (WT), lactate production-deficient mutant (LM), and chromosomal integrant of LM with an enhanced 3P4::pta asRNA module (LM3P). (A) Cell growth was profiled with an insert table showing the growth rate of each strain under the tested condition. Product titers in the end-point fermentation broth were measured at the end fermentation with $5 \mathrm{~g} / \mathrm{L}$ cellobiose (B) or $10 \mathrm{~g} / \mathrm{L}$ cellulose (C). Error bar represents the standard deviation of three biological replicates. 
expression thereby reducing acetate production in these strains. Combined utilization of gene repression and Cas9 nickase genome editing realized a one-step markerless integration of an upgraded antisense RNA-expressing module into the chromosome, genetically allowing stable manipulation of essential genes and providing a technical demonstration of the unmatched editing simplicity and efficiency of this system over double-crossover recombination. The tandem promoter strategy dramatically improved repression of acetate formation in the integrants. This combinatorial strategy significantly expanded our ability to manipulate more diverse genes for functional characterization and strain engineering.

\section{AUTHOR CONTRIBUTIONS}

TX and YL designed the study and performed the experiments. TX drafted the manuscript. JVN helped data visualization and revised the manuscript. $\mathrm{ZH}$ and $\mathrm{JZ}$ participated in the conception of the study, helped data interpretation and revised

\section{REFERENCES}

Andersch, W., Bahl, H., and Gottschalk, G. (1983). Level of enzymes involved in acetate, butyrate, acetone and butanol formation by Clostridium acetobutylicum. Eur. J. Appl. Microbiol. Biotechnol. 18, 327-332. doi: 10.1007/ Bf00504740

Argyros, D. A., Tripathi, S. A., Barrett, T. F., Rogers, S. R., Feinberg, L. F., Olson, D. G., et al. (2011). High ethanol titers from cellulose by using metabolically engineered thermophilic, anaerobic microbes. Appl. Environ. Microbiol. 77, 8288-8294. doi: 10.1128/AEM.00646-11

Barrangou, R. (2015). Diversity of CRISPR-Cas immune systems and molecular machines. Genome Biol. 16, 247. doi: 10.1186/s13059-015-0816-9

Bikard, D., Jiang, W. Y., Samai, P., Hochschild, A., Zhang, F., and Marraffini, L. A. (2013). Programmable repression and activation of bacterial gene expression using an engineered CRISPR-Cas system. Nucleic Acids Res. 41, 7429-7437. doi: 10.1093/nar/gkt520

Brown, S. D., Guss, A. M., Karpinets, T. V., Parks, J. M., Smolin, N., Yang, S., et al. (2011). Mutant alcohol dehydrogenase leads to improved ethanol tolerance in Clostridium thermocellum. Proc. Natl. Acad. Sci. U.S.A. 108, 13752-13757. doi: 10.1073/pnas.1102444108

Cheung, J. K., Awad, M. M., McGowan, S., and Rood, J. I. (2009). Functional analysis of the VirSR phosphorelay from Clostridium perfringens. PLoS ONE 4:e5849. doi: 10.1371/journal.pone.0005849

Desai, R. P., and Papoutsakis, E. T. (1999). Antisense RNA strategies for metabolic engineering of Clostridium acetobutylicum. Appl. Environ. Microbiol. 65, 936-945.

Desvaux, M. (2005). Clostridium cellulolyticum: model organism of mesophilic cellulolytic clostridia. FEMS Microbiol. Rev. 29, 741-764. doi: 10.1016/j.femsre. 2004.11.003

Desvaux, M., Guedon, E., and Petitdemange, H. (2000). Cellulose catabolism by Clostridium cellulolyticum growing in batch culture on defined medium. Appl. Environ. Microbiol. 66, 2461-2470.

Friehs, K. (2004). Plasmid copy number and plasmid stability. Adv. Biochem. Eng. Biotechnol. 86, 47-82.

Gao, F., and Zhang, R. R. (2011). Enzymes are enriched in bacterial essential genes. PLOS ONE 6:e21683. doi: 10.1371/journal.pone.00 21683

Georg, J., and Hess, W. R. (2011). cis-Antisense RNA, another level of gene regulation in bacteria. Microbiol. Mol. Biol. Rev. 75, 286-300. doi: 10.1128/ Mmbr.00032-10

Glass, J. I., Assad-Garcia, N., Alperovich, N., Yooseph, S., Lewis, M. R., Maruf, M., et al. (2006). Essential genes of a minimal bacterium. Proc. Natl. Acad. Sci. U.S.A. 103, 425-430. doi: 10.1073/pnas.0510013103 the manuscript. All authors read and approved the final manuscript.

\section{FUNDING}

This work was supported by the NSF EPSCoR Program through the award EPS 0814361.

\section{SUPPLEMENTARY MATERIAL}

The Supplementary Material for this article can be found online at: http://journal.frontiersin.org/article/10.3389/fmicb. 2017.01744/full\#supplementary-material

FIGURE S1 | RNA structures of targeted transcript regions of pta $(\mathbf{A}, \mathbf{B})$ and ack (C,D) predicted by RNAfold web server. The color represents base-pair probabilities. (A,C) Secondary structure with minimal free energy; (B,D) centroid secondary structure.

Gruber, A. R., Lorenz, R., Bernhart, S. H., Neuboock, R., and Hofacker, I. L. (2008). The Vienna RNA websuite. Nucleic Acids Res. 36, W70-W74. doi: 10.1093/nar/ gkn188

Guedon, E., Desvaux, M., and Petitdemange, H. (2002). Improvement of cellulolytic properties of Clostridium cellulolyticum by metabolic engineering. Appl. Environ. Microbiol. 68, 53-58.

Heap, J. T., Ehsaan, M., Cooksley, C. M., Ng, Y. K., Cartman, S. T., Winzer, K., et al. (2012). Integration of DNA into bacterial chromosomes from plasmids without a counter-selection marker. Nucleic Acids Res. 40, e59. doi: 10.1093/nar/gkr1321

Hemme, C. L., Fields, M. W., He, Q., Deng, Y., Lin, L., Tu, Q. C., et al. (2011). Correlation of genomic and physiological traits of Thermoanaerobacter species with biofuel yields. Appl. Environ. Microbiol. 77, 7998-8008. doi: 10.1128/Aem. 05677-11

Higashide, W., Li, Y., Yang, Y., and Liao, J. C. (2011). Metabolic engineering of Clostridium cellulolyticum for production of isobutanol from cellulose. Appl. Environ. Microbiol. 77, 2727-2733. doi: 10.1128/AEM.02454-10

Ji, Y. D., Marra, A., Rosenberg, M., and Woodnutt, G. (1999). Regulated antisense RNA eliminates alpha-toxin virulence in Staphylococcus aureus infection. J. Bacteriol. 181, 6585-6590.

Juhas, M., Eberl, L., and Church, G. M. (2012). Essential genes as antimicrobial targets and cornerstones of synthetic biology. Trends Biotechnol. 30, 601-607. doi: 10.1016/j.tibtech.2012.08.002

Kung, S. H., Retchless, A. C., Kwan, J. Y., and Almeida, R. P. (2013). Effects of DNA size on transformation and recombination efficiencies in Xylella fastidiosa. Appl. Environ. Microbiol. 79, 1712-1717. doi: 10.1128/AEM.03525-12

Lee, J. H., Sung, B. H., Kim, M. S., Blattner, F. R., Yoon, B. H., Kim, J. H., et al. (2009). Metabolic engineering of a reduced-genome strain of Escherichia coli for L-threonine production. Microb. Cell Fact. 8:2. doi: 10.1186/1475-2859-8-2

Lee, S. Y., Mermelstein, L. D., and Papoutsakis, E. T. (1993). Determination of plasmid copy number and stability in Clostridium acetobutylicum ATCC-824. FEMS Microbiol. Lett. 108, 319-323. doi: 10.1016/0378-1097(93)90562-G

Levine, E., Zhang, Z., Kuhlman, T., and Hwa, T. (2008). Correction: quantitative characteristics of gene regulation by small RNA. PLoS Biol. 6:e5. doi: 10.1371/ journal.pbio.0060005

Li, Y., Tschaplinski, T. J., Engle, N. L., Hamilton, C. Y., Rodriguez, M. Jr, Liao, J. C., et al. (2012). Combined inactivation of the Clostridium cellulolyticum lactate and malate dehydrogenase genes substantially increases ethanol yield from cellulose and switchgrass fermentations. Biotechnol. Biofuels 5:2. doi: 10.1186/1754-6834-5-2

Li, Y., Xu, T., Tschaplinski, T. J., Engle, N. L., Yang, Y., Graham, D. E., et al. (2014). Improvement of cellulose catabolism in Clostridium cellulolyticum by sporulation abolishment and carbon alleviation. Biotechnol. Biofuels 7:25. doi: $10.1186 / 1754-6834-7-25$ 
Lin, P. P., Mi, L., Morioka, A. H., Yoshino, K. M., Konishi, S., Xu, S. C., et al. (2015). Consolidated bioprocessing of cellulose to isobutanol using Clostridium thermocellum. Metab. Eng. 31, 44-52. doi: 10.1016/j.ymben.2015.07.001

Luo, M. L., Leenay, R. T., and Beisel, C. L. (2016). Current and future prospects for CRISPR-based tools in bacteria. Biotechnol. Bioeng. 113, 930-943. doi: 10.1002/ bit. 25851

Lynd, L. R., van Zyl, W. H., McBride, J. E., and Laser, M. (2005). Consolidated bioprocessing of cellulosic biomass: an update. Curr. Opin. Biotechnol. 16, 577-583. doi: 10.1016/j.copbio.2005.08.009

Man, S. A., Cheng, R. B., Miao, C. C., Gong, Q. H., Gu, Y. C., Lu, X. Z., et al. (2011). Artificial trans-encoded small non-coding RNAs specifically silence the selected gene expression in bacteria. Nucleic Acids Res. 39, e50. doi: 10.1093/nar/gkr034

Martinez-Alonso, M., Garcia-Fruitos, E., Ferrer-Miralles, N., Rinas, U., and Villaverde, A. (2010). Side effects of chaperone gene co-expression in recombinant protein production. Microb. Cell Fact. 9:64. doi: 10.1186/14752859-9-64

Na, D., Yoo, S. M., Chung, H., Park, H., Park, J. H., and Lee, S. Y. (2013). Metabolic engineering of Escherichia coli using synthetic small regulatory RNAs. Nat. Biotechnol. 31, 170-174. doi: 10.1038/nbt.2461

Papanek, B., Biswas, R., Rydzak, T., and Guss, A. M. (2015). Elimination of metabolic pathways to all traditional fermentation products increases ethanol yields in Clostridium thermocellum. Metab. Eng. 32, 49-54. doi: 10.1016/j. ymben.2015.09.002

Perret, S., Maamar, H., Belaich, J. P., and Tardif, C. (2004). Use of antisense RNA to modify the composition of cellulosomes produced by Clostridium cellulolyticum. Mol. Microbiol. 51, 599-607. doi: 10.1046/j.1365-2958.2003. 03860.x

Pfaffl, M. W. (2001). A new mathematical model for relative quantification in real-time RT-PCR. Nucleic Acids Res. 29, e45.

Qi, L. S., Larson, M. H., Gilbert, L. A., Doudna, J. A., Weissman, J. S., Arkin, A. P., et al. (2013). Repurposing CRISPR as an RNA-guided platform for sequencespecific control of gene expression. Cell 152, 1173-1183. doi: 10.1016/j.cell.2013. 02.022
Rose, I. A. (1955). Acetate kinase of bacteria (acetokinase). Methods Enzymol. 1, 591-595. doi: 10.1016/0076-6879(55)01102-6

Shaw, A. J., Podkaminer, K. K., Desai, S. G., Bardsley, J. S., Rogers, S. R., Thorne, P. G., et al. (2008). Metabolic engineering of a thermophilic bacterium to produce ethanol at high yield. Proc. Natl. Acad. Sci. U.S.A. 105, 13769-13774. doi: 10.1073/pnas.0801266105

Thomason, M. K., and Storz, G. (2010). Bacterial antisense RNAs: how many are there, and what are they doing? Annu. Rev. Genet. 44, 167-188. doi: 10.1146/ annurev-genet-102209-163523

Wang, B., and Kuramitsu, H. K. (2005). Inducible antisense RNA expression in the characterization of gene functions in Streptococcus mutans. Infect. Immun. 73, 3568-3576.

Xu, T., Li, Y., Shi, Z., Hemme, C. L., Zhu, Y., Van Nostrand, J. D., et al. (2015). Efficient genome editing in Clostridium cellulolyticum via CRISPRCas9 nickase. Appl. Environ. Microbiol. 81, 4423-4431. doi: 10.1128/AEM. 00873-15

Xu, T., Li, Y. C., Van Nostrand, J. D., He, Z. L., and Zhou, J. Z. (2014). Cas9-based tools for targeted genome editing and transcriptional control. Appl. Environ. Microbiol. 80, 1544-1552. doi: 10.1128/Aem.03786-13

Zhu, Y., Liu, X., and Yang, S. T. (2005). Construction and characterization of pta gene-deleted mutant of Clostridium tyrobutyricum for enhanced butyric acid fermentation. Biotechnol. Bioeng. 90, 154-166. doi: 10.1002/bit.20354

Conflict of Interest Statement: The authors declare that the research was conducted in the absence of any commercial or financial relationships that could be construed as a potential conflict of interest.

Copyright (C) $2017 \mathrm{Xu}, \mathrm{Li}, \mathrm{He}$, Van Nostrand and Zhou. This is an open-access article distributed under the terms of the Creative Commons Attribution License (CC BY).

The use, distribution or reproduction in other forums is permitted, provided the original author(s) or licensor are credited and that the original publication in this journal is cited, in accordance with accepted academic practice. No use, distribution or reproduction is permitted which does not comply with these terms. 